\title{
Good Things Still Come in Old Packages: Cryosurgery vs LEEP
}

Milestones in the management of cervical intraepithelial neoplasia (CIN) and cervical cancer include the introduction of routine cytologic screening programs and colposcopy. These tools promoted early detection and proper evaluation of cervical lesions, which often allowed treatment by cryosurgery and laser ablation rather than surgical conization or hysterectomy. The loop electrosurgical excision procedure (LEEP) was later hailed as another great advancement in the treatment of CIN, but for many it became a two-edged sword.

It was not that long ago that the abnormal $\mathrm{Pa}$ panicolaou smear was a rare occurrence. In the 1970s, when residents would ask me the correct approach to the evaluation of a class III Papanicolaou smear, I would scratch my head and remember that 6 months earlier we had encountered a similar situation and had sent the patient to the gynecologist for conization. In the late 1970 s and early 1980s, when colposcopy made its appearance, clinicians rejoiced that we no longer had to perform a conization on all patients with high-grade lesions. Once invasive cancer was excluded, the cervix could be spared. General anesthesia could be eliminated in many cases. Most often, cryotherapy and, later, laser ablation would resolve the disease process.

Although laser technology was considered to be superior by many, in comparative studies with properly selected patients, cryotherapy was just as efficacious. Two recent review studies compare the cryosurgery data well. ${ }^{1,2}$ Except for large highgrade lesions, in cases where conization was truly indicated, or where there was concurrent extensive disease in the vagina or in the vulva, laser proved to be only a more' expensive way of treating CIN.

The point should not be missed that the purpose of colposcopy is to evaluate the cervix and determine whether it requires treatment or observation. Rather than performing a conization on every cervix with a high-grade lesion, we were now able to select patients for proper, limited treatment. Re-

Submitted 17 June 1999.

From The National Procedures Institute, Midland, Mich. Reprints not available from the author. moving excessive amounts of the cervix was no longer necessary in everyone. A milestone, indeed!

When the LEEP procedure was introduced in 1991, two approaches were initially available. The first was to evaluate the condition of the cervix and treat the lesion on the first visit. In other words, the patient with an abnormal Papanicolaou smear had further evaluation with colposcopy, and if any abnormality was seen, LEEP was carried out during the same visit. This approach led to a great many women who underwent the procedure unnecessarily. ${ }^{3}$ It is now only in rare instances that the seeand-treat method is advised. Rather, it is recommended that colposcopic assessment be carried out and that the cervix be treated based on the visual and histologic findings. ${ }^{4,5}$ The purpose again is to save cervical tissue and not overtreat.

A main argument against cryotherapy has been that cryotherapy drives the squamocolumnar junction into the os and makes further colposcopic assessments inadequate, which then leads to a conization should the patient have further abnormalities. In other words, cryotherapy should be avoided because it could make colposcopic assessments afterward more difficult. Curiously, what has become openly advocated instead is that the LEEP procedure be performed for the treatment of all high-grade cervical intraepithelial neoplasia in nearly every case because the squamocolumnar junction remains on the external os and will be clearly visible for future evaluations. The fallacy of this logic appears obvious. It says that we need to give everyone a conization (a LEEP procedure is, indeed, conization, whether it is shallow or deep) to prevent the possibility of having a future conization because of the effects of the treatment of cryotherapy! Those of us who do LEEP procedures also realize that many times the squamocolumnar junction is also shifted into the canal after LEEP, so compared with cryotherapy, little is gained.

The article by Stienstra and colleagues ${ }^{6}$ in this issue of The fournal clearly refutes the initial assertion that cryotherapy drives the squamocolumnar junction into the canal, thus making future colposcopic assessment inadequate. This study might 
seem to be rather unimportant, but the possible implications are huge. It removes a barrier to the return of cryotherapy as the preferred method of treatment CIN if criteria are met.

Based on three resources, ${ }^{7-9}$ the 50 th percentile of physician fees for cervical cryotherapy in the country is $\$ 210$ compared with $\$ 836$ for the LEEP procedure. Often there are charges for a surgical tray, because use of disposable loops, pad, handpieces, and so on, can add nearly $\$ 100$ to the procedure. In addition, there is the pathologist's fee for reading the LEEP specimen. In our hospital, for a single excision and endocervical curettage, the total hospital and professional fee would be $\$ 364$. If a "cowboy hat" second excision is done, the total laboratory fee would be $\$ 480$. Amazingly to me, many LEEP procedures are still performed in the operating room with the patient given general anesthesia, which can add another $\$ 3000$ to costs! Without a doubt, the LEEP procedure is six to eight times more costly than cryotherapy if performed in the office and 20 to 22 times more expensive if performed in the operating room.

The beauty of the LEEP procedure is that it is simple and well tolerated. The danger of the procedure is that it removes more tissue, is overused, and is expensive when the alternative of cryotherapy is an equally effective treatment in many instances. A recent study by Mitchell et al ${ }^{10}$ shows that cryotherapy, laser, and the loop procedure, in properly selected patients, have similar complications, persistence, and recurrence rates. These outcomes are also similar for high-grade lesions (yes, for CIN III and carcinoma in situ). The common factor for recurrence is the size of the lesion. The American College of Obstetricians and Gynecologists (ACOG) committee opinion bulletin states, "There appears to be no valid reason for proscribing or limiting the use of cryocautery in the treatment of CIN."11

I am uncertain why the study by Stienstra et al shows only a $76 \%$ resolution for CIN. Other studies report resolution in the $90 \%$ range except for large or deep lesions. ${ }^{1,2}$ Their study does not define whether resolution was by histologic biopsy or by colposcopic visualization. Reparative changes persist much longer after cryotherapy than after other excisional procedures and can be confusing colposcopically and when viewed microscopically. Were warty changes or warty atypia included in the failures? Are these really failures? If the Papanicolaou smear was positive, was that included as a documented failure? Clearly the purpose of this article was not to document the efficacy of cryotherapy; efficacy has been confirmed in numerous previous studies of large magnitude.

The importance of the findings of Stienstra et al are that cryotherapy does not preclude an adequate colposcopic assessment afterward and does not automatically relegate the patient to a conization procedure should there be recurrent disease. The ACOG committee bulletin opinion even suggests consideration of a second cryotherapy where treatment is indicated. ${ }^{11}$ Practically speaking, however, if we have a failure of cryotherapy, most of us would probably proceed to a LEEP procedure if a high-grade lesion persisted.

Could the flawed logic of doing a LEEP procedure on everyone with CIN be influenced by the level of physician reimbursement? Some argue to use LEEP for all high-grade lesions for tissue confirmation and medicolegal documentation of the extent of disease. But is that what we are about as physicians? Should our goal not be to preserve as much of the cervix as is safely possible? Are we not to be patient advocates? ${ }^{12}$ Should we not be rejoicing that we have effective treatment methods such as cryotherapy, which removes 3 to $5 \mathrm{~mm}$ of tissue, compared with LEEP, which removes 8 to $18 \mathrm{~mm}$ of tissue? Should we not be concerned about the high cost of medicine regardless of whether we are in a managed care environment? Should we not critically ask the questions, Where is the proof that LEEP is superior to cryotherapy? If cryotherapy has documented effectiveness for 25 years, why all of a sudden did it lose its effectiveness when the LEEP procedure was introduced?

The article by Mitchell et $\mathrm{al}^{10}$ shows that the preferred method of treatment for CIN would be cryotherapy because of its low cost, low complication rate, efficacy, lack of complications, and the preservation of cervical tissue. Others concur. ${ }^{13-16}$ The loop procedure might be fun to perform and well received by patients, but it is more expensive, removes more cervix, and is not the preferred choice for treatment of most CIN.

Conclusions by Stienstra et al then remove one more argument from the armamentarium of those advocating LEEP for the treatment of all highgrade CIN including small focal lesions. Family physicians need not believe they are providing inferior, out-of-date care by using cryotherapy in 
properly selected patients. Indeed, it is to be considered the treatment of choice unless otherwise contraindicated. If physician reimbursements were the same, and there was a diagnostic-related group for treating CIN, more use of cryotherapy would be evident.

John L. Pfenninger, MD

Midland, Mich

\section{References}

1. Burke, L. Evolution of therapeutic approaches to cervical intraepithelial neoplasia. J Lower Genital Tract Dis 1997;1:267-73.

2. Ferris DG. Cryotherapy of the cervix. J Lower Genital Tract Dis 1998;2:98-105.

3. Ferris DG, Hainer BC, Pfenninger JL, Zuber TJ. "See and treat" electrosurgical loop excision of the cervical transformation zone. J Fam Pract 1996;42: 253-7.

4. Ang MS, Kaufman RH, Adam E, et al. Colposcopically directed biopsy and loop excision of the transformation zone. Comparison of histologic findings. $J$ Reprod Med 1995;40:167-70.

5. Kurman RJ, Henson DE, Herbst AL, Noller KL, Schiffman MH. Interim guidelines for management of abnormal cervical cytology. The 1992 National Cancer Institute Workshop. JAMA 1994;271: 1866-9.

6. Stienstra KA, Brewer BE, Franklin LA. A comparison of flat and shallow conical tips for cervical cryotherapy. J Am Board Fam Pract 1999;12:000-00.
7. 1999 Physician's fee \& coding guide. Sixth annual edition. Augusta, Ga: Health Care Consultants of America, 1995.

8. Physician's fee reference 1999. $16^{\text {th }}$ ed. West Allis, Wisc: Yale Wasserman, DMP Medical Publishers, 1999.

9. 1999 National fee analyzer: pricing guide for evaluating fees nationwide. Salt Lake City: Medicode, 1998.

10. Mitchell MF, Tortolero-Luna G, Cook E, Whittaker L, Rhodes-Morris H, Silva E. A randomized clinical trial of cryotherapy, laser vaporization, and loop electrosurgical excision for treatment of squamous intraepithelial lesions of the cervix. Obstet Gynecol 1998;92:737-44.

11. Use of cryotherapy in the treatment of cervical intraepithelial neoplasia. ACOG committee opinion, No 34. ACOG Committee on Gynecologic Practice. Washington, DC: ACOG, 1985, revised 1988.

12. Pfenninger JL. Colposcopy, LEEP and other procedures: the role for family physicians. Fam Med 1996; 28:505-7.

13. Noller KL. Cryotherapy, laser vaporization, and loop electrosurgical excision for treatment of squamous intraepithelial lesions of the cervix. Comment. OB/GYN Clin Alert 1998;Dec:61.

14. Noller KL. Colposcopy: the evolution of a technique. Colposcopist 1994;26(4):2.

15. Ferris DG. Electrosurgical loop excision of the cervical transformation zone: preclinical training proficiency. Fam Med 1993;25:456-60.

16. News. J Natl Cancer Inst 1998;90:351-3. 